\title{
Quantitative genetics of intraspecies hybrids
}

\author{
IAN L. GORDON* \\ Institute of Molecular BioSciences, Massey University, Private Bag 11222, Palmerston North, New Zealand
}

\begin{abstract}
Quantitative genetics generally is based on the properties of the randomly fertilized (RF) population or inbred derivatives of it. Simple hybrids and hybrid swarms do not conform to this model; and only some properties of hybrid means appear to have been available. In this paper, several genetical properties are derived, including genotype and allele frequencies, genotypic variance, broad-sense heritability, and outbreeding coefficient. The earlier mean is confirmed, and hybrid vigour is examined critically. These results make it possible to evaluate quantitatively both natural selection and forward selection (in plant breeding) from hybrids. An important finding is that hybrids with maximum hybrid vigour do not maximize genetic advance from forward selection, i.e. evolution is unlikely to enhance hybrid vigour. Another finding is that the concepts of additive genetic variance and narrow-sense heritability are inappropriate for hybrids, owing to the genetic disequilibrium inherent from their origin, and to the ephemeral nature of their population structure.
\end{abstract}

Keywords: genetic advance, genetic variances, heritability, hybrids, outbreeding, selection.

\section{Introduction}

The majority of quantitative genetics is based on the properties of the disomic randomly fertilized population, either as a single panmictic gamodeme in equilibrium or as a dispersed inbred derivative of it (e.g. Wright, 1951, 1952; Crow \& Kimura, 1970; Mather \& Jinks, 1971; Falconer, 1981). The fundamental quantitative genetic concepts and details are defined with respect to such populations, and include most of the common facts: allele and genotype frequencies, population mean, genotypic variances, heritabilities, selection genetic advance and effects of inbreeding. However, there are other types of populations which are common in nature and in plant breeding for which these properties may not apply. These populations include hybrids ( $F_{1}$ 'hybrid swarms' and simple crosses), and segregating $\mathrm{F}_{2}$ bulks of either sibcrossed or selfed origin. These are, intrinsically, in disequilibrium by virtue of their very origin. The basic properties arising from the randomly fertilized population (RF) generally may not apply to them. In order to evaluate this, and to account for the effects of such populations in evolution and in plant breeding, it is necessary to reveal their quantitative genetic properties.

The present paper examines the quantitative genetic properties of the bulk hybrid produced by unhindered crossing between two parent populations. ${ }^{1}$ Previous

\footnotetext{
*E-mail: genovir@massey.ac.nz

${ }^{1}$ Impediments to gene flow, such as species incompatibility, maternal restitution, transgenic instability, organelle dosage, etc., are not considered in this paper.
}

work has explored the mean of such an $F_{1}$, having been stimulated by interest in hybrid vigour (Falconer, 1981). Also, some knowledge on the genotype frequencies within hybrids is widely extant, but only for the simple case of a hybrid between two complementary pure lines (homozygotes) (Wright, 1952; Kempthorne, 1956). Here, all of the properties mentioned earlier are derived from first principles, including an outbreeding coefficient. The main novel information from this paper is the definition of the hybrid's genotypic variance $\left(\sigma_{\mathrm{G}}^{2}\right)$, and broad-sense heritability $\left(h_{\mathrm{B}}^{2}\right)$. We will discover that the genic variance $\left(\sigma_{\mathrm{A}}^{2}\right)$ and dominance variance $\left(\sigma_{\mathrm{D}}^{2}\right)$ cannot be defined, and are inappropriate for hybrids.

\section{General method}

The classical two-allele population gene-model is used (e.g. Falconer, 1981), where $p=$ frequency of $A_{1}$ (the better allele-expectation, or allele), and $q=$ frequency of $A_{2}$ (the lesser allele-expectation, or allele), without any omission (i.e. $p+q=1$ ).

The genotype effects are deviates from the homozygote midpoint (MP), where $a$ is the $A_{1} A_{1}$ homozygote effect (an expectation), $-a$ is the $A_{2} A_{2}$ effect, and $d$ is the heterozygote effect. The row vector of these effects is $\mathbf{g}^{\prime}=[a, d,-a]$. Notice that this is a biometrical model and not a single-gene model. In this form, it describes either the net results (expectations) of polygenic systems (Mather \& Jinks, 1971; Jana, 1972), or the sensu strictu single-gene case. It is a single main-effect model only, 
and does not attempt to define epistasis as interactions amongst factorial main effects. Some forms of epistasis may be accommodated by overdominance $(d>a)$. Although this is a simplification, it is a standard approach which has served quantitative genetics well for many decades (Mather \& Jinks, 1971; Falconer, 1981).

Alternative models, with factorial main effects and interactions, fall into two categories: biometrical expectations over many loci (Cockerham, 1954; Kempthorne, 1956; Hayman, 1958), or sensu strictu polygene specifications (Seyffert, 1966; Jana, 1971). The latter refer to specified loci situations, such as transgenics, mutants, or simply inherited specific phenotypes; they are not considered in this paper. For this initial examination of the properties of the hybrid, we will use the classical, robust, biometrical simple main-effects model which is the foundation of quantitative genetics.

In most of the examples used in this paper, $a=10$ and $d=7.5$ (a case of partial dominance with $d<a$ ). When overdominance $(d>a)$ is being considered, the following values of $d$ are substituted: 15, 22.5 and 30. (In my experience, biometrical overdominance is extremely rare in practice, and allowing for a case of $d=3 a$ probably exceeds any reality.)

One random-fertilizing parent population $\left(\mathrm{P}_{1}\right)$ is regarded as focal, with frequencies $p_{1}$ and $q_{1}$. The other random-fertilizing parent population $\left(\mathrm{P}_{2}\right)$ is defined as an offset from this where $p_{2}=p_{1}-y$, and $q_{2}=q_{1}+y$, with $y=p_{1}-p_{2}$. This follows the procedure of Falconer (1981), and defines the two parental populations within a single system. This is intrinsically better than Kempthorne's approach (Kempthorne, 1956) which is incapable of revealing the interconnectedness arising from hybridization, i.e. the disequilibrium (y). As $p_{1} \rightarrow 1$ and $p_{2} \rightarrow 0$ (or vice versa) with $y \rightarrow \pm 1$, a simple hybrid between two complementary pure lines is defined.

The methods used actually to derive the hybrid properties form an integral part of the Results of this paper, and are presented in that section.

It is often instructive to compare the $F_{1}$ with a random-fertilizing population $(\mathrm{RF})$ based on $p_{\mathrm{F} 1}$. The properties of such a population are obtained in the usual way (see, e.g. Falconer, 1981) after incorporating the new allele frequency into the equations:

$\sigma_{\mathrm{A}(\mathrm{RF})}^{2}=2 p_{\mathrm{F} 1} q_{\mathrm{F} 1} \alpha_{\mathrm{F} 1}^{2} \quad\left(\right.$ where $\alpha_{\mathrm{F} 1}=a+\left(\begin{array}{ll}q_{\mathrm{F} 1} & \left.\left.p_{\mathrm{F} 1}\right) d\right),\end{array}\right.$

and

$\sigma_{\mathrm{D}(\mathrm{RF})}^{2}=\left(2 p_{\mathrm{F} 1} q_{\mathrm{F} 1}\right)^{2} d^{2}$.

\section{Results}

The frequencies of progeny genotypes following hybridization are obtained as the products between the two parental arrays of allele frequencies $\left[p_{1}, q_{1}\right]$ and $\left[p_{1}-y\right.$, $\left.q_{1}+y\right]$, assuming that each parent is contributing equally to the cross. Thus, we are considering the biparental cross (BiP) and hybrid-cultivar in plant breeding; and, in evolution, the mean bidirectional hybrid between two populations. The gamete union is presented in Table 1.

These results lead readily to the following progeny genotype frequencies (row vector $\mathbf{f}^{\prime}$ ):

$$
\begin{array}{ll}
A_{1} A_{1} & \left(\mathbf{f}_{11}\right)=p_{1}^{2} \quad p_{1} y \\
A_{1} A_{2} & \left(\mathbf{f}_{12}\right)=2 p_{1} q_{1}+y\left(p_{1} \quad q_{1}\right) \\
A_{2} A_{2} & \left(\mathbf{f}_{22}\right)=q_{1}^{2}+q_{1} y .
\end{array}
$$

As discussions of hybrids often focus on their heterozygosity, we examine $\mathbf{f}_{12}$ in Fig. 1, where complementary opposite crosses (i.e. $p_{2}=1-p_{1}$ ) are shown,

Table 1 Gamete fertilization outcomes and frequencies between two hybridizing populations

\begin{tabular}{llllll}
\hline \multirow{2}{*}{$\begin{array}{l}\text { Other } \\
\text { parent }\left(\mathrm{P}_{2}\right)\end{array}$} & \multicolumn{4}{c}{ Focal parent $\left(\mathrm{P}_{1}\right)$} \\
\cline { 2 - 5 } & $A_{1}$ & $p_{1}$ & $A_{2}$ & $q_{1}$ \\
\hline$A_{1}$ & $p_{1}-y$ & $A_{1} A_{1}$ & $\left(p_{1}-y\right) p_{1}$ & $A_{1} A_{2}$ & $\left(p_{1}-y\right) q_{1}$ \\
$A_{2}$ & $q_{1}+y$ & $A_{2} A_{1}$ & $\left(q_{1}+y\right) p_{1}$ & $A_{2} A_{2}$ & $\left(q_{1}+y\right) q_{1}$ \\
\hline
\end{tabular}

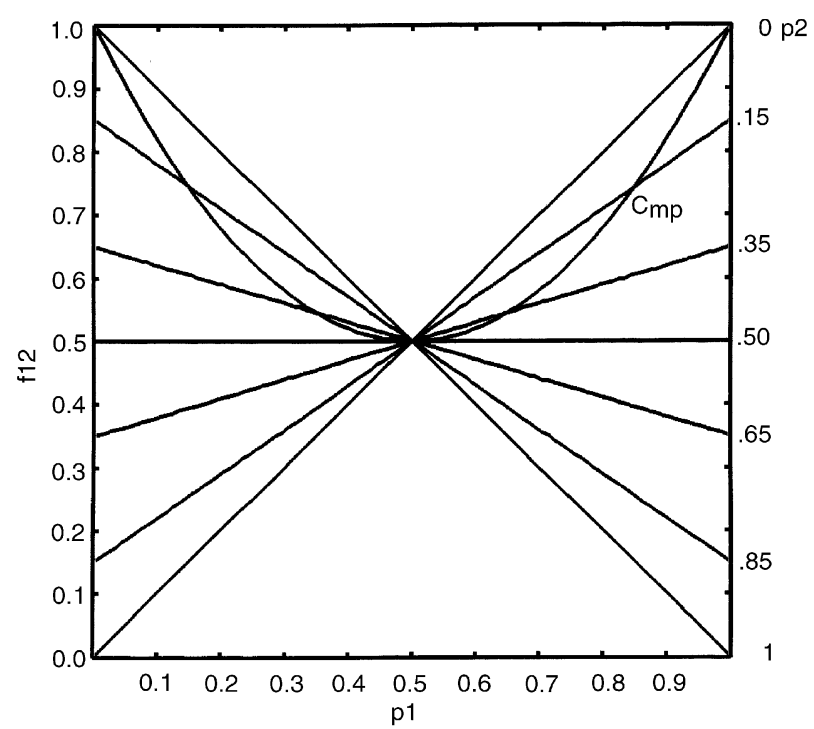

Fig. 1 Frequencies of heterozygotes in the $\mathrm{F}_{1}\left(\mathbf{f}_{12}\right)$ from crosses between the focus parent $\left(A_{1}\right.$ frequency $\left.p_{1}\right)$ and the other parent with various fixed $p_{2}$. The complementary-opposite other parent is compared also.

(c) The Genetical Society of Great Britain, Heredity, 83, 757-764. 
together with a range of fixed other parents (i.e. $p_{2}=0$, $0.15,0.35,0.5,0.65,0.85$ or 1$)$. Notice that a fully heterozygous hybrid occurs only when opposite pure lines $\left(p_{1}=1\right.$ and $p_{2}=0$, or vice versa) are crossed.

\section{$F_{1}$ allele frequency}

The hybrid's $A_{1}$ 'allele' (allele-expectation or actual allele) frequency $\left(p_{\mathrm{F} 1}\right)$ is found as the weighted mean of the progeny $\left(F_{1}\right)$ genotype frequencies $\mathbf{f}^{\prime}$. The weights reflect the proportions of the focus 'allele' in each genotype in the $\mathrm{F}_{1}$, i.e. for $A_{1}$ the weights are $\mathbf{w}_{1}^{\prime}=[1,1 / 2,0]$ for the $\mathrm{F}_{1}$ genotypes $\left\{A_{1} A_{1}, A_{1} A_{2}\right.$ and $\left.A_{2} A_{2}\right\}$, respectively. Similarly, for $q_{\mathrm{F} 1}$, the frequency of $A_{2}$, the weights are $\mathbf{w}_{2}^{\prime}=[0,1 / 2,1]$ instead. Thus,

$$
\begin{aligned}
p_{\mathrm{F} 1}= & \mathbf{w}_{1}^{\prime} \mathbf{f} \\
= & 1\left(p_{1}^{2} \quad p_{1} y\right)+\frac{1}{2}\left(2 p_{1} q_{1}+y\left(p_{1} \quad q_{1}\right)\right) \\
& +0\left(q_{1}^{2}+q_{1} y\right) \\
= & p_{1} \quad \frac{1}{2} y .
\end{aligned}
$$

Similarly, the new $A_{2}$ frequency is:

$$
\begin{aligned}
q_{\mathrm{F} 1} & =\mathbf{w}_{2}^{\prime} \mathbf{f} \\
& =q_{1}+\frac{1}{2} y .
\end{aligned}
$$

The frequency $p_{\mathrm{F} 1}$ is examined in Fig. 2 for the complementary-opposite crosses mentioned earlier, and

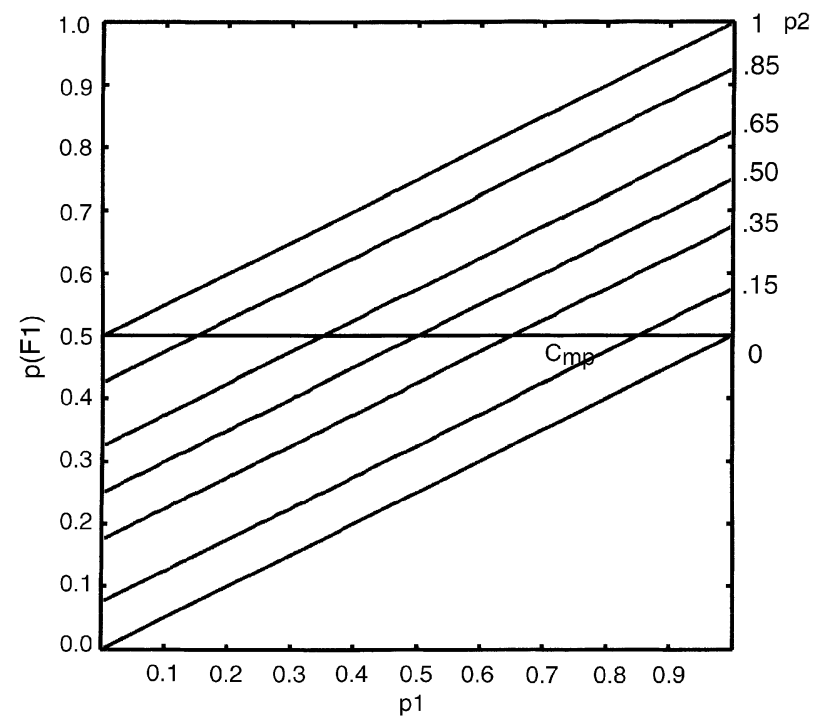

Fig. 2 Frequencies of the $A_{1}$ allele in the $\mathrm{F}_{1}\left(p_{\mathrm{F} 1}\right)$ of crosses between the focus parent ( $A_{1}$ frequency $p_{1}$ ), and the other parent either with complementary-opposites $p_{2}$ or with various fixed $p_{2}$. the previous series of fixed other-parents. Notice that complementary-opposite crosses always result in a hybrid allele frequency of 0.5 , as do several other combinations of $p_{1}$ and $p_{2}$. In general, by rearranging the previous equations, the parental frequency required to obtain any nominated $p_{\mathrm{F} 1}$ can be estimated from either $p_{1}=2 p_{\mathrm{F} 1}-p_{2}$, or $p_{2}=2 p_{\mathrm{F} 1}-p_{1}$, depending on which values are provided and which are sought. For the special case of $p_{\mathrm{F} 1}=1 / 2$ these reduce to $p_{1}=1-p_{2}$ and $p_{2}=1-p_{1}$, respectively.

\section{Hybrid mean}

The $\mathrm{F}_{1}$ mean $\left(\bar{F}_{1}\right)$ is $\mu+\bar{G}_{\mathrm{F} 1}$, where $\mu$ is the attribute background mean, and $\bar{G}_{\mathrm{F} 1}(=\gamma$ also $)$ is the gene-model mean. This is obtained as follows, using the vectors defined earlier.

$$
\begin{aligned}
& \bar{G}_{\mathrm{F} 1}=\mathbf{f}^{\prime} \mathbf{g} \\
& =\left(\begin{array}{ll}
p_{1}^{2} & \left.p_{1} y\right) a+\left(2 p_{1} q_{1}+y\left(p_{1} \quad q_{1}\right)\right) d
\end{array}\right. \\
& +\left(q_{1}^{2}+q_{1} y\right)(a) \\
& =\left[\begin{array}{ll}
p_{1} & \left.q_{1}\right) a+2 p_{1} q_{1} d
\end{array}\right] \\
& y\left[a+\left(\begin{array}{ll}
q_{1} & p_{1}
\end{array}\right) d\right] \\
& =\bar{G}_{1} \quad y \alpha_{1} \text {. }
\end{aligned}
$$

Here, $\alpha_{1}$ is the well known average-allele substitution effect for the focal parent population (see, e.g. Falconer, 1981), being equal to $a+\left(q_{1}-p_{1}\right) d$.

This is the same result as obtained by Falconer (1981), but the derivation here is much more direct. The value of this $\bar{F}_{1}$ is explored in Fig. 3 for complementaryopposite crosses and moderate partial dominance $(a=10, d=7.5$ and $\mu=10)$. It is useful to compare it in Fig. 3 with the equivalent means of both $\mathrm{P}_{1}$ and $\mathrm{P}_{2}$, the parental mean $(P M)$, and with the RF with $p_{\mathrm{F} 1}=0.5$. (In order to save space, we will cease illustrating with fixed $p_{2}$ crosses from here on.)

Notice that the $F_{1}$ mean increases as $p_{1} \rightarrow 0$ or $p_{1} \rightarrow 1$, but it is never greater than the mean of the better parent (which is $\mathrm{P}_{2}$ when $p_{1}<1 / 2$ and $\mathrm{P}_{1}$ when $\left.p_{1}>1 / 2\right)$ in this example showing partial dominance.

Changes in dominance (particularly overdominance, in which $d>a$ ) elevate the mean considerably, especially as $p_{1} \rightarrow 0$ or 1 .

A common understanding of 'hybrid vigour' is that the 'hybrid is more vigorous than the parents' - i.e. both parents. The formal definition does not say that, however, as it defines vigour with respect to the mid-parent (i.e. the parental mean, $P M$ ). The common hybrid vigour definition is $h v=\bar{F}_{1} \quad P M$ (Falconer, 1981), who also shows that $h v=y^{2} d$ in terms of the gene model. This is a very optimistic view of hybrid vigour, because 


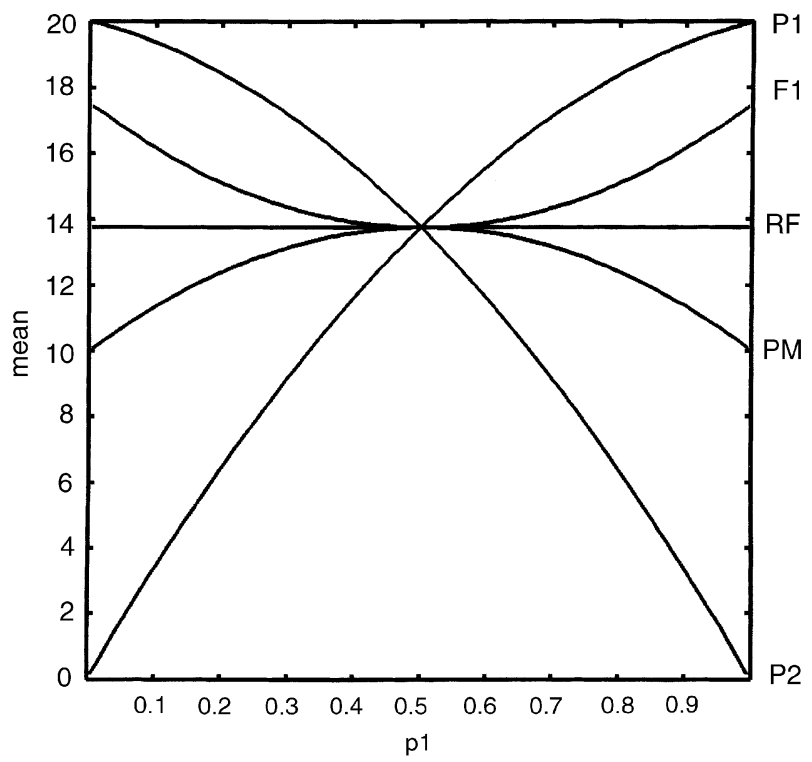

Fig. 3 Population mean of the $\mathrm{F}_{1}$ of crosses between the focus parent $\left(A_{1}\right.$ frequency $\left.p_{1}\right)$ and the other parent with complementary-opposite $p_{2}$. The two parent means $\left(\mathrm{P}_{1}\right.$ and $\left.\mathrm{P}_{2}\right)$ are shown for comparison, along with the bi-parental mean (PM) and RF mean for $p_{\mathrm{F} 1}=1 / 2 .(a=10, d=7.5, \mu=10$. $)$

midparent is never more than the mean of the better parent. An alternative view (hybrid vigour (b): Mather \& Jinks, 1971) is more in line with the common concept of hybrid vigour, and is defined as follows:

$h b=\bar{F}_{1} \quad \bar{P}^{\max }, \quad$ where $\bar{P}^{\max }={ }^{\max }\left\{\bar{P}_{1}, \bar{P}_{2}\right\}$.

When $p_{1}>p_{2}$, parent $\mathrm{P}_{1}$ will be the better parent. We can then derive hybrid vigour (b) $(h b)$ in terms of the gene model as follows:

$$
\begin{aligned}
& h b=\bar{F}_{1} \quad \bar{P}_{1} \\
& \left.=\left[\begin{array}{lll}
a\left(p_{1}\right. & q_{1} & y
\end{array}\right)+d\left(2 p_{1} q_{1} \quad y\left(q_{1} \quad p_{1}\right)\right)\right] \\
& {\left[a\left(p_{1} \quad q_{1}\right)+2 p_{1} q_{1} d\right]} \\
& =y\left(a+d\left(\begin{array}{ll}
q_{1} & p_{1}
\end{array}\right)\right) \\
& =y \alpha_{1} \text {. }
\end{aligned}
$$

With $\mathrm{P}_{1}$ as the better parent, $y$ is positive: so $h b$ will be positive (i.e. real) only when $\alpha_{1}$ is negative. The definition of $\alpha_{1}$ shows that this will occur when $p_{1} d>\left(a+q_{1} d\right)$. As $y$ becomes negative, it means that $\mathrm{P}_{2}$ is now the better parent, and we simply swap our definition base to $h b=-y \alpha_{2}$. In Fig. 4 , the levels of $h b$ from several degrees of dominance are examined. Hybrids with partial dominance do not express hybrid vigour (b); nor do any hybrids with central values of $p_{1}$. Vigour maximizes as $p_{1} \rightarrow 0$ or 1 , and as overdominance increases.

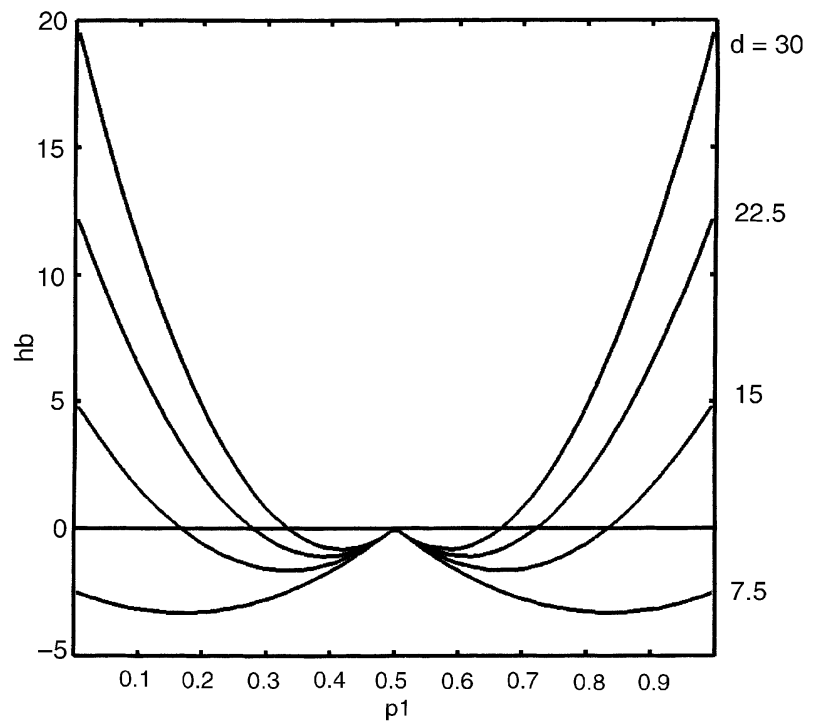

Fig. 4 Heterosis from better parent (hb) in hybrids between a focus parent $\left(A_{1}\right.$ frequency $\left.p_{1}\right)$ and the other parent with complementary $p_{2}$. Using $a=10$, partial dominance has $d=7.5$; while overdominance increases as $d=15,22.5$ and 30 .

\section{Hybrid genotypic variance}

The genotypic variance of a hybrid population has the usual expectation:

$\sigma_{\mathrm{G}}^{2}=\sum_{i \leq i^{\prime}}^{3} f_{i i^{\prime}} g_{i i^{\prime}}^{2} \quad \gamma^{2}$,

where the first right-hand-side term is an unadjusted sum of squares (USS) and the second term is a correction factor $(\mathrm{CF})^{2}$. The expanded components are as follows.

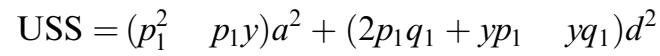

$$
\begin{aligned}
& +\left(q_{1}^{2}+q_{1} y\right)(a)^{2} \text {. } \\
& \left.\mathrm{CF}=\left[\begin{array}{lll}
p_{1} & q_{1} & y
\end{array}\right) a+\left(2 p_{1} q_{1} \quad y\left(q_{1} \quad p_{1}\right)\right) d\right]^{2} \\
& =p_{1}^{2} a^{2} \quad 2 p_{1} q_{1} a^{2}+q_{1}^{2} a^{2}+4 p_{1}^{2} q_{1} a d \quad 4 p_{1} q_{1}^{2} a d \\
& +4 p_{1}^{2} q_{1}^{2} d^{2}+y^{2} a^{2}+2 y^{2} q_{1} a d \quad 2 y^{2} p_{1} a d+y^{2} p_{1}^{2} d^{2} \\
& y^{2} 2 p_{1} q_{1} d^{2}+y^{2} q_{1}^{2} d^{2}+2 y q_{1} a^{2} \quad 2 y p_{1} a^{2}+2 y p_{1}^{2} a d \\
& 2 y 4 p_{1} q_{1} a d+2 y q_{1}^{2} a d+2 y 2 p_{1}^{2} q_{1} d^{2} \quad 2 y 2 p_{1} q_{1}^{2} d^{2} .
\end{aligned}
$$

\footnotetext{
${ }^{2}$ This sum of squares is based on frequencies rather than counts: so it is a mean-square in fact.
}

(c) The Genetical Society of Great Britain, Heredity, 83, 757-764. 
After subtracting the CF from the USS and effecting several gathering and factoring of terms ${ }^{3}$, the sum of squares becomes:

$$
\begin{aligned}
& \operatorname{SS}\left(\sigma_{\mathrm{G}_{\mathrm{F} 1}}^{2}\right) \\
& =2 p_{1} q_{1} a^{2}+\left(\begin{array}{ll}
q_{1} & p_{1}
\end{array}\right) 4 p_{1} q_{1} a d+2 p_{1} q_{1} d^{2}\left(\begin{array}{ll}
1 & 2 p_{1} q_{1}
\end{array}\right)
\end{aligned}
$$

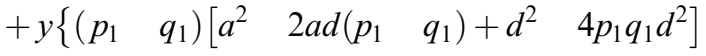

$$
\begin{aligned}
& \left.\left.+2 p_{1} q_{1} 2 a d\right\} \quad y^{2}\left[\begin{array}{ll}
a^{2}+\left(q_{1}\right. & p_{1}
\end{array}\right) 2 a d+\left(\begin{array}{ll}
q_{1} & p_{1}
\end{array}\right)^{2} d^{2}\right] \\
& =2 p_{1} q_{1}\left[a^{2}+\left(\begin{array}{ll}
q_{1} & p_{1}
\end{array}\right) 2 a d+\left(\begin{array}{ll}
q_{1} & p_{1}
\end{array}\right)^{2} d^{2}\right] \\
& +2 p_{1} q_{1}\left(2 p_{1} q_{1} d^{2}\right)+y\left\{( \begin{array} { l l } 
{ p _ { 1 } } & { q _ { 1 } }
\end{array} ) \left[\begin{array}{l}
a^{2}+\left(\begin{array}{ll}
q_{1} & p_{1}
\end{array}\right) 2 a d \\
p_{1}
\end{array}\right.\right. \\
& \left.\left.+d^{2}\left(1 \quad 4 p_{1} q_{1}\right)\right]+4 p_{1} q_{1} a d\right\} \quad y^{2} \alpha_{1}^{2} \\
& =2 p_{1} q_{1} \alpha_{1}^{2}+\left(2 p_{1} q_{1}\right)^{2} d^{2}+y\left(p_{1} \quad q_{1}\right) \alpha_{1}^{2} \quad y^{2} \alpha_{1}^{2}+y 4 p_{1} q_{1} a d \text {. }
\end{aligned}
$$

Recalling the classical definitions of $\sigma_{\mathrm{A}}^{2}$ and of $\sigma_{\mathrm{D}}^{2}$ (Falconer, 1981), and defining $\operatorname{cov}(a, d)=4 p q a d$, we can write:

$$
\begin{aligned}
\sigma_{\mathrm{G}_{\mathrm{F} 1}}^{2}= & \sigma_{\mathrm{A}_{1}}^{2}\left(1+\frac{y\left(p_{1}\right.}{\left.2 q_{1}\right)} \frac{y^{2}}{2 p_{1} q_{1}} \quad \frac{2 p_{1} q_{1}}{)}\right) \\
& +\sigma_{\mathrm{D}_{1}}^{2}+y \operatorname{cov}(a, d)_{1} .
\end{aligned}
$$

The overall genotypic variance is visualized for our partial dominance and complementary-opposites cross in Fig. 5. There, it is compared also with the genotypic variance of the focal parent $\left(\mathrm{P}_{1}\right)$, and of the $\mathrm{RF}$ population based on $p_{\mathrm{F} 1}=1 / 2$ as before.

Notice that simple hybrids between opposite pure lines have no genotypic variance for the gene effect in question, as one would expect. For $p_{1}<1 / 2$, the $F_{1}$ has smaller genotypic variance than both the focal parent and the equivalent RF. For $p_{1}>1 / 2$, its variance lies between that of the other two populations. This pattern is true even for overdominance: which greatly inflates the total amount of genotypic variance in all three population types.

The genic and dominance variances of hybrid populations present a problem of definition, and even of existence. Therefore they will be addressed in the Discussion rather than here in the Results.

\footnotetext{
${ }^{3}$ Various relations are used to resolve these equations, and are given below.

$\left(p^{2}-2 p q+q^{2}\right)=$ either $(p-q)^{2}$ or $(q-p)^{2}$;

$(1-2 p q)=p^{2}-2 p q+q^{2}+2 p q=(q-p)^{2}+2 p q$;

$(1-4 p q)=(q-p)^{2}$

$-2 a d(p-q)=(+) 2 a d(q-p)$.
}

Also, recall that $\alpha=a+(q-p) d$ (e.g. Falconer, 1981).

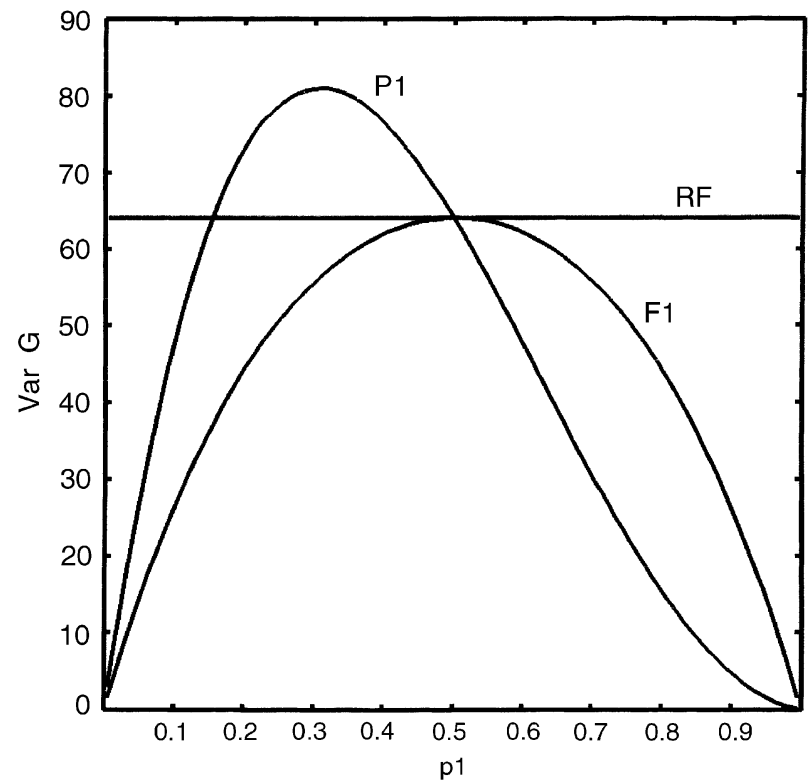

Fig. 5 Genotypic variance (Var G) of hybrids between a focus parent $\left(A_{1}\right.$ frequency $\left.p_{1}\right)$ and the other parent with complementary-opposite $p_{2}$. Partial dominance $(a=10, d=7.5)$ is shown. Variance of the focal parent $\left(\mathrm{P}_{1}\right)$ and of the RF for $p_{\mathrm{F} 1}=1 / 2$ are compared.

\section{Heritability}

Having defined a genotypic variance for the $F_{1}$, we have a corresponding ratio of $\sigma_{\mathrm{G}}^{2}$ to $\sigma_{\mathrm{P}}^{2}$ : that is, a broad-sense heritability. As genic variance is undefined, there is no narrow-sense heritability. The broad-sense heritability $\left(h_{\mathrm{B}}^{2}\right)$ is a biometrical parameter, it being the genotypic determination of the phenotype (Wright, 1951). This broad-sense heritability would therefore be appropriate for obtaining the genetic advance from forward selection from hybrids. It is shown in Fig. 6, using the various parameter values given elsewhere, and an example environmental variance of $\sigma_{\mathrm{E}}^{2}=25$.

Note that the broad-sense heritability attains maximum values for a wide range of hybrids with $p_{1}$ of central values, and increases with increasing dominance (d). We would expect therefore to see a maximal result from forward selection in such hybrids, with a minimal response in hybrids between extreme parents (i.e. complementary-opposite parents, and $p_{1} \rightarrow 0$ or 1 ). This matter raises interesting discussion (later) about the relative merits of hybrid vigour and selection.

\section{Outbreeding coefficient}

The definition is based on the level of heterozygosity in the hybrid as contrasted to a relevant reference population: in this case a RF population with allele frequency $p_{\mathrm{F} 1}$. It is as follows: 


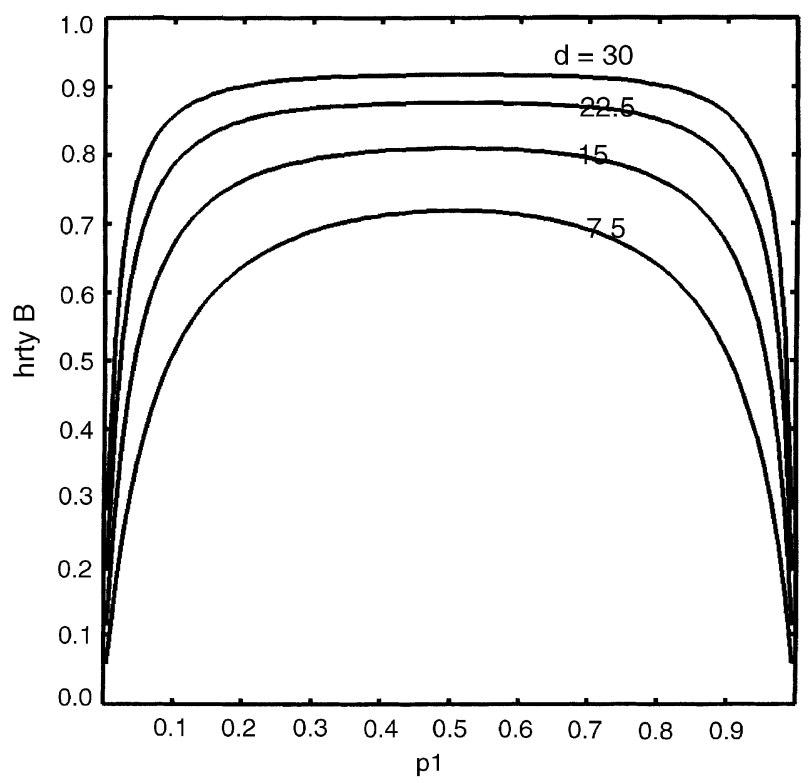

Fig. 6 Broad-sense heritability $\left(h_{\mathrm{B}}^{2}\right)$ under four levels of dominance $(d=7.5,15,22.5,30)$ for hybrids between a focus parent $\left(A_{1}\right.$ frequency $\left.p_{1}\right)$ and the other parent with complementary-opposite $p_{2} .\left(a=10, \sigma_{\mathrm{E}}^{2}=25\right)$.

$\phi=\left(\begin{array}{ll}1 & \frac{f_{12_{\mathrm{F} 1}}}{f_{12 \mathrm{RF}}}\end{array}\right)$

where all symbols have been defined previously.

This is also one approach to defining an inbreeding coefficient: but in this case the higher levels of heterozygosity in the $\mathrm{F}_{1}$ make it negative. This means it indicates 'new heterozygosity relative to a RF population', i.e. outbreeding. Its values for complementary-opposites crosses, and for fixed $P_{2}$ crosses $\left(p_{2}=0\right.$, $0.5,1)$ are shown in Fig. 7. For the complementaryopposites, outbreeding maximizes (that is $\phi \rightarrow-1$ ) for extreme values of $y\left(p_{1} \rightarrow 0\right.$ or $\left.p_{1} \rightarrow 1\right)$ : in other words, the proportion of heterozygotes approaches twice that of the comparable RF population. When $p_{1}=p_{2}=1 / 2$, the hybrid has the same heterozygosity as the corresponding RF population, and the outbreeding coefficient is zero.

The situations for fixed parents are more variable. Three special cases are shown in Fig. 7. First, for $p_{2}=0.5$, the curve shape across $p_{1}$ is similar to that for complementary-opposite crosses, with the exception that $\phi_{\min }=-0.325$ rather than -1 . That is, the level of heterozygosity is never more than a third higher than in the reference RF population. The other two special cases involve the two pure lines ( $p_{2}=0$ or 1 ). In each case, the level of outbreeding changes from -1 (full heterozygosity) to 0 ( $\mathrm{RF}$ heterozygosity) as the focus parent and the other parent change from being complementary-opposite

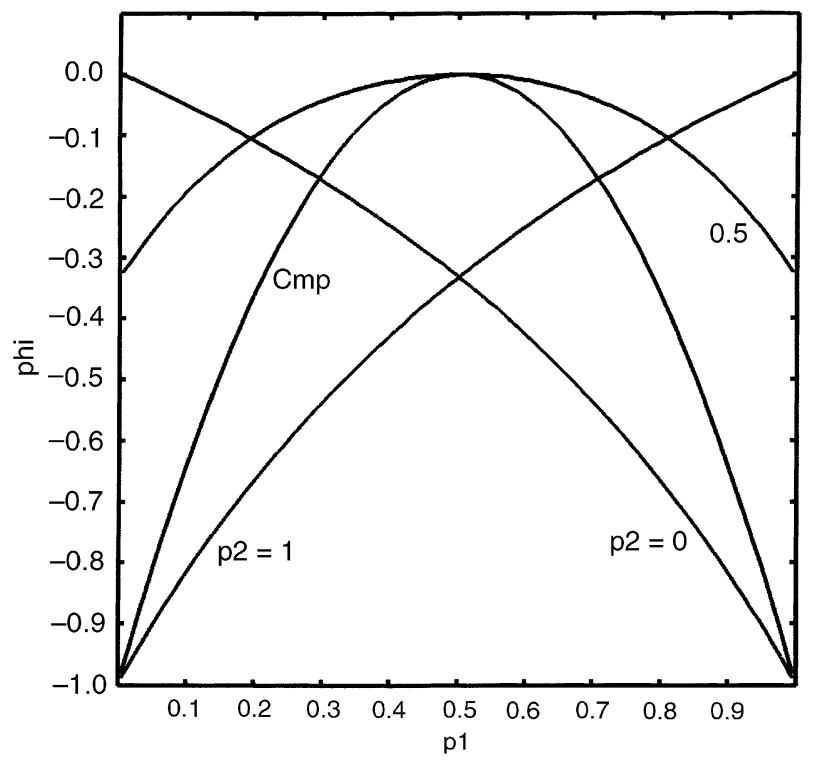

Fig. 7 Outbreeding coefficient (phi $(\phi))$ for hybrids between a focus parent ( $A_{1}$ frequency $p_{1}$ ) and the other parent, with $p_{2}=0,1,0.5$ and complementary-opposite.

to nearly identical. At the point of both parents being the same pure lines $\left(p_{1}=p_{2}=p_{\mathrm{F} 1}=0\right.$, or 1$)$, there is no heterozygosity in any of the three populations, and outcrossing is zero. ${ }^{4}$ (The term ' $\mathrm{F}_{1}$ ' is, of course, somewhat forced at this juncture.)

\section{Discussion}

\section{Genic and dominance variances in the $F_{1}$}

It is well-known that the classical RF genotypic variance contains the two components: genic and dominance variances. From eqn (1), it is obvious that the $F_{1}$ genotypic variance has a different composition. As the hybrid population structure is sexually ephem$\mathrm{eral}^{5}$, the very concept of average allele substitution is questionable. This means that the $\sigma_{\mathrm{A}}^{2}$ component (eqn ?tul $>1$ ) should not be interpreted as the $F_{1}$ genic variance, because such average allele effect is the very basis of such a variance (Falconer, 1981). It is simply a function of the focal parent's genic variance which forms part of the definition of the $F_{1}$ genotypic variance. Furthermore, the $F_{1}$ genotypic variance (eqn 1) also contains a third component excess $\operatorname{cov}(a, d)$ - which does not appear in the

\footnotetext{
${ }^{4}$ The graph covers only the range $p_{1}=0.005-0.995$ in order to avoid division by zero in the computer algorithm.

${ }^{5}$ Following $F_{1}$ meiosis and syngamy, it no longer contains the same genotypic frequencies with which it began: it is not in equilibrium. In fact, it produces a new kind of population, the $F_{2}$.
} 
Table 2 Genetic advance $\left(\Delta_{\mathrm{G}}\right)$ at selection pressure of $10 \%$ $(P=0.1)$, variability $\left(\sigma_{\mathrm{P}}^{2}\right)$, broad-sense heritability $\left(h_{\mathrm{B}}^{2}\right)$, and mean of the $\mathrm{F}_{1}$ from complementary-opposites crosses for various focus parents (allele frequency $p_{1}$ ) and two dominance levels $(d=7.5,30$ with $a=10)$

\begin{tabular}{lcrrcc}
\hline$d$ & $p_{1}$ & \multicolumn{1}{c}{$\Delta_{\mathrm{G}}$} & \multicolumn{1}{c}{$\sigma_{\mathrm{P}}^{2}$} & $h_{\mathrm{B}}^{2}$ & $\mathrm{~F}_{1}$ mean \\
\hline 7.5 & 0.35 & 11.34 & 84.389 & 0.704 & 14.09 \\
& 0.45 & 11.84 & 88.498 & 0.718 & 13.79 \\
& 0.97 & 2.68 & 33.894 & 0.202 & 17.06 \\
30.0 & 0.35 & 27.49 & 293.409 & 0.915 & 26.35 \\
& 0.45 & 27.81 & 299.203 & 0.916 & 25.15 \\
& 0.97 & 10.80 & 80.096 & 0.688 & 38.25 \\
\hline
\end{tabular}

classical RF case. ${ }^{6}$ These observations remind us that a hybrid is profoundly different from a RF population. Therefore, the concepts of genic and dominance variances appear inappropriate with respect to hybrids.

\section{Selection efficiency}

Several of the properties (heritability, genotypic variance, mean) have the very important implication that some hybrids will be more efficient for subsequent selection than others. Obviously, those with $h_{\mathrm{B}}^{2} \rightarrow 0$ will be of little value for forward selection (i.e. selecting $F_{1}$ individuals towards an $\mathrm{F}_{2}$ nursery). ${ }^{7}$ Likewise, those with trivial $\sigma_{\mathrm{G}}^{2}$ will be of little utility, because $\sigma_{\mathrm{P}}^{2} \rightarrow \sigma_{\mathrm{E}}^{2}$. In the case of our illustrative complementary-opposites crosses (see Figs 5 and 6), hybrids between 'extremely opposite' parents (i.e. $p_{1}<0.2$ or $p_{1}>0.8$ ) are undesirable if forward selection is the intention, especially where overdominance prevails. These properties are illustrated in Table 2 , where genetic advance $\left(\Delta_{\mathrm{G}}\right)$ at selection pressure of $10 \%(P=0.1)$ in selecting $\mathrm{F}_{1} \rightarrow \mathrm{F}_{2}$ is given, for partial and overdominance $(d=7.5$ and 30$)$

\footnotetext{
${ }^{6}$ The RF genotypic variance can be derived biometrically, in a manner similar to that adopted here for the $F_{1}$. Indeed, Mather \& Jinks (1971) must surely have done so previously, but it never seems to have been published overtly. By that approach, it can be shown that the original $\mathrm{RF} \quad \sigma_{\mathrm{A}}^{2}=\sigma_{\mathrm{a}}^{2}+\left(\begin{array}{ll}q & p\end{array}\right) \operatorname{cov}(a, d)+\left(\begin{array}{ll}q & p\end{array}\right)^{2} \sigma_{\mathrm{d}}^{2}=2 p q \alpha^{2}$, where $\sigma_{\mathrm{a}}^{2}=$ $2 p q a^{2}$ (the gene-model homozygote variance), $\sigma_{\mathrm{d}}^{2}=2 p q d^{2}$ (the genemodel heterozygote variance), and other terms are defined previously. For this classical RF situation, all of the covariance is utilized in the absorption into that genic variance. This contrasts strongly with the situation we have revealed for the hybrid.

The so-called dominance variance in the RF population is simply the remnant $\sigma_{\mathrm{d}}^{2}$ which could not be absorbed into the genic variance. In fact, $\sigma_{\mathrm{D}}^{2}=2 p q \sigma_{\mathrm{d}}^{2}=(2 p q)^{2} d^{2}$.

${ }^{7} \Delta_{\mathrm{G}(\mathrm{F} 1)}=i h_{\mathrm{B}}^{2} \sigma_{\mathrm{P}}$, where $i=$ the standardized selection differential (accounting for selection pressure, P), and other symbols have been defined elsewhere in this paper (and in Falconer, 1981).
}

and complementary-opposite crosses, with parental inputs which are intermediate $\left(p_{1}=0.35\right)$, optimal $\left(p_{1}=0.45\right)$, and extreme $\left(p_{1}=0.97\right)$. Background properties are given also, such as hybrid $\sigma_{\mathrm{P}}^{2}$ and $h_{\mathrm{B}}^{2}$.

All of the trends discussed above are borne out by these values. Notice that the greatest $\Delta_{\mathrm{G}}$ occurs at the $p_{1}=0.45$ example within each dominance level; whereas the highest hybrid mean occurs at $p_{1}=0.97$ within each dominance level. The drop in $\sigma_{\mathrm{P}}^{2}$ for extreme $p_{1}$ is conspicuous; and reduced $h_{\mathrm{B}}^{2}$ for extreme $p_{1}$ is apparent. These hybridization outcomes affect natural selection as well, i.e. adaptation is optimized out of hybrid swarms between nonextreme parental populations, rather than those arising from extreme opposites.

For backward selection, ${ }^{8}$, matters are completely different. The relevant property of the hybrid is the mean (or more specifically, its hybrid vigour), which is higher towards extreme ends of the $p_{1}$ range (Fig. 3). As we are here using the hybrid's mean performance as a progeny test of the parents, and not selecting $F_{1}$ individuals onwards into the next generation, heritability is not an issue (except insofar as it is biometrically related to the standard error of the mean). So, in this case, the 'most extreme opposites' crosses would be the best, just as Falconer (1981) declared when he stated that $y$ should be maximized to maximize hybrid vigour. The last column of Table 2 shows this for the illustrative example. This aspect of using hybrids appears to be entirely anthropomorphic: it is very unlikely to apply to evolution because retrospective re-making of particular crosses requires a discerning control of the programme. Also, we have shown that hybrids which maximize hybrid vigour do not maximize forward genetic advance, indicating that natural selection does not operate to maximize hybrid vigour.

\section{References}

COCKerham, C. C. 1954. Extension of the concept of partitioning hereditary variance for analysis of covariances among relatives when epistasis is present. Genetics, 39, 859-882.

CROW, J. F. AND KIMURA, M. 1970. An Introduction to Population Genetics Theory. Harper \& Row, New York.

FALCONER, D. S. 1981. An Introduction to Quantitative Genetics, 2nd edn. Longman, New York.

HAYMAN, B. I. 1958. Separation of epistatic from additive and dominance variation in generation means. Heredity, 12, 371-390.

${ }^{8}$ i.e. using the hybrid means to identify which parents have given good hybrid combinations of genes as expressed in their progeny (the $F_{1}$ ). The hybrid mean performance is a backward-looking progeny test. Parents identified as having good hybrid combining ability would then be re-crossed to propagate the desirable hybrid. 
JANA, S. 1971. Simulation of quantitative characters from qualitatively acting genes. I. Non-allelic gene interactions involving two or three loci. Theor. Appl. Genet., 41, 216-226.

JANA, S. 1972. Biometrical analysis with two or three gene loci. Can. J. Genet. Cytol., 14, 31-38.

KEMPTHORNE, O. 1956. The theory of the diallel cross. Genetics, 41, 451-459.

MATHER, K. AND JINKS, J. L. 1971. Biometrical Genetics, 2nd edn. Chapman \& Hall, London.
SEYFFERT, w. 1966. Die Simulation quantitativer Merkmale durch Gene mit biochemisch definierbarer Wirkung. I. Ein einfaches Modell. Züchter, 36, 159-163.

Wright, s. 1951. The genetical structure of populations. Ann. Eugen., 15, 323-354.

WRIGHT, S. 1952. The genetics of quantitative variability. In: Reeve, E. C. R. and Waddington, C. H. (eds) Quantitative Inheritance, pp. 5-41. Agricultural Research Council, London. 